\title{
Bosentan in the treatment of pulmonary arterial hypertension with the focus on the mildly symptomatic patient
}

This article was published in the following Dove Press journal:

Vascular Health and Risk Management

29 July 2009

Number of times this article has been viewed

\section{Christopher J Valerio John G Coghlan}

Department of Cardiology, Royal Free Hospital, London, UK
Correspondence: Christopher JValerio Pulmonary Hypertension Office, Department of Cardiology, Royal Free Hospital, Pond Street, Hampstead, London, UK NW3 2QG Tel +44 (0) 2077940500 Ext 33648 Fax +44 (0) 2078302428

Email cjvalerio@doctors.net.uk
Abstract: Pulmonary arterial hypertension $(\mathrm{PAH})$ is a progressive disease with poor survival outcomes. Bosentan is an oral endothelin-1 receptor antagonist (ERA) that has been shown in a large randomized placebo-controlled trial (BREATHE-1) to be effective at improving exercise tolerance in patients with PAH in functional class III and IV. Further studies have been conducted showing: benefit in smaller subgroups of PAH, eg, congenital heart disease, efficacy in combination with other PAH therapies, eg, sildenafil, improved long-term survival compared with historical controls. More recently, controlled trials of new ERAs have included patients with milder symptoms; those in functional class II. Analysis of the functional class II data is often limited by small numbers. These trials have generally shown a similar treatment effect to bosentan, but there are no controlled trials directly comparing these new ERAs. The EARLY trial exclusively enrolled functional class II patients and assessed hemodynamics at 6 months. Though significant, the reduction in pulmonary vascular resistance is merely a surrogate marker for the intended aim of delaying disease progression. Significant adverse effects associated with bosentan include edema, anemia and transaminase elevation. These may preclude a long duration of treatment. Further studies are required to determine optimum treatment strategy in mild disease.

Keywords: pulmonary arterial hypertension, bosentan, endothelin-1 receptor antagonist

\section{Introduction to the management of pulmonary arterial hypertension}

Pulmonary hypertension is a broad term which refers to elevated pressure in the pulmonary arterial tree. There are several mechanisms by which this can occur and this is reflected in the Venice classification (see Table 1). ${ }^{1}$ Pulmonary arterial hypertension (PAH) is the term used in the presence of changes which directly affect the pulmonary vessels, ie, group 1 pulmonary hypertension which is the concern of this review. This includes a seemingly diverse group of diseases, but the underlying patho-physiology is thought to be similar: vasoconstriction, smooth muscle cell and endothelial proliferation, and intravascular thrombosis. ${ }^{2}$ An updated classification is awaited from the expert meeting at Dana Point in 2008, but the make-up of groups 1-5 will not be significantly altered.

The gold standard diagnostic test is a right heart catheter study and the criteria for diagnosis are: mean pulmonary artery pressure (mPAP) greater than $25 \mathrm{mmHg}$ at rest or $30 \mathrm{mmHg}$ with exercise, pulmonary capillary wedge pressure less than or equal to $15 \mathrm{mmHg}$ and a pulmonary vascular resistance (PVR) of greater than or equal to 240 dynes $/ \mathrm{s} / \mathrm{cm}^{5} .{ }^{1}$ The diagnosis of pulmonary hypertension is often delayed and requires a thorough assessment to exclude other pathologies and 
Table I The Venice classification of pulmonary hypertension 2003

Group I. Pulmonary arterial hypertension

I.I. Idiopathic pulmonary arterial hypertension

I.2. Familial pulmonary arterial hypertension

I.3. Pulmonary arterial hypertension associated with:

I.3.I. Collagen vascular disease, eg, scleroderma, systemic lupus erythematosus, rheumatoid arthritis

I.3.2. Congenital systemic-to-pulmonary shunts

I.3.3. Portal hypertension, eg, ethanol induced cirrhosis

I.3.4. HIV infection

1.3.5. Drugs and toxins, eg, fenfluramine

I.3.6. Other (thyroid disorders, glycogen storage disease, Gaucher's disease, hereditary hemorrhagic telangiectasia, hemoglobinopathies, myeloproliferative disorders, splenectomy)

I.4. Associated with significant venous or capillary involvement

I.4. I. Pulmonary veno-occlusive disease (PVOD)

I.4.2. Pulmonary capillary hemangiomatosis $(\mathrm{PCH})$

I.5. Persistent pulmonary hypertension of the newborn

\section{Group 2. Pulmonary hypertension with left heart disease}

2. I. Left sided atrial or ventricular heart disease

2.2. Left sided valvular heart disease

Group 3. Pulmonary hypertension associated with lung disease or hypoxemia

3.I. Chronic obstructive pulmonary disease

3.2. Interstitial lung disease

3.3. Sleep-disordered breathing

3.4. Alveolar hypoventilation disorders

3.5. Chronic exposure to high altitude

3.6. Developmental abnormalities

Group 4. Pulmonary hypertension due to chronic thrombotic, embolic disease, or both

4.I.Thromboembolic obstruction of proximal pulmonary arteries

4.2. Thromboembolic obstruction of distal pulmonary arteries

4.3. Non-thrombotic pulmonary embolism (tumor, parasites or foreign material)

\section{Group 5. Miscellaneous}

Eg, sarcoidosis, pulmonary Langerhans'-cell histiocytosis, lymphangiomatosis, granulomatous disease, compression of pulmonary vessels (adenopathy, tumor or fibrosing mediastinitis)

Adapted with permission from Simonneau G, Galiè N, Rubin LJ, et al. Clinical classification of pulmonary hypertension. J Am Coll Cardiol. 2004;43 Suppl S: 5S-I2S. Copyright (C) 2004. Elsevier.

identify the probable cause of pulmonary hypertension. Vaso-reactivity challenge is important to identify those patients who will benefit from calcium channel blockers. ${ }^{3}$ Cardio-pulmonary exercise testing is used at some centers and may be helpful to identify those patients with exercise-induced pulmonary hypertension. ${ }^{4}$ It is important to exclude chronic thrombo-embolic disease with ventilation-perfusion scanning. ${ }^{5}$ Screening programs using echocardiography are recommended for groups at high risk of developing PAH: first-degree relatives of patients with idiopathic $\mathrm{PAH}$, people with known genetic mutations for PAH, scleroderma, congenital heart disease with systemic-to-pulmonary shunts and portal hypertension being considered for liver transplantation. ${ }^{3}$ More information on further investigations and determining the type of pulmonary hypertension can be found in recognized guidelines. $3,5,6$

Once a diagnosis of PAH is established there are several assessments widely used to monitor progress. The use of the World Health Organization (WHO) modified functional classification (FC) scale (Table 2) allows for standardized grading, which is incorporated into treatment guidelines. ${ }^{3}$ The six-minute walk distance (6MWD) is frequently utilized in trials of PAH therapy as the primary endpoint. It is an appealing measure because of its simplicity and replication 
Table 2 Functional classification of pulmonary hypertension modified after the NYHA functional classification according to the World Health Organization

Class I - Patients with pulmonary hypertension but without resulting limitation of physical activity. Normal physical exertion does not cause undue dyspnea or fatigue, chest pain or near syncope.

Class II - Patients with pulmonary hypertension resulting in slight limitation of physical activity. They are comfortable at rest. Ordinary physical activity causes undue dyspnea or fatigue, chest pain or near syncope.

Class III - Patients with pulmonary hypertension resulting in marked limitation of physical activity. They are comfortable at rest. Less than ordinary physical activity causes undue dyspnea or fatigue, chest pain or near syncope.

Class IV - Patients with pulmonary hypertension with inability to carry out any physical activity without symptoms. These patients manifest signs of right heart failure. Dyspnea and/or fatigue may even be present at rest. Discomfort is increased by any physical activity.

of the dominant clinical feature of cardio-respiratory disease - reduced exercise ability - but also carries prognostic significance. ${ }^{7}$ Additionally oxygen saturations can be measured at peak exercise, and those patients whose levels fall below $90 \%$ may benefit from ambulatory oxygen. The Borg dyspnea index is a visual analogue score which attempts to quantify effort during the 6MWD. General health-related questionnaires, such as short form survey 36 and specific ones, such as the Camphor questionnaire, are available tools.

Several general measures are recommended for PAH. Firstly, where there is an associated cause, eg, sickle cell anemia, optimization of this condition is recommended. It is noteworthy that pulmonary vasculopathy associated with systemic lupus erythematosis and mixed connective tissue disease may respond to immunosuppression. ${ }^{8,9}$ Lifestyle advice includes limiting exercise to avoid symptoms, family planning advice and advance planning if surgery or anesthesia are required. ${ }^{6}$ Oxygen therapy is appropriate in the presence of hypoxemia and should also be considered for those undertaking air travel as the low cabin pressure may precipitate breathlessness. Because of the high risk of intravascular thrombosis in the small pulmonary arterioles, anticoagulation with warfarin is recommended. For patients in whom right heart failure has developed, diuretics are indicated to offload excess fluid. Digoxin is often prescribed for its inotropic effect. Calcium channel blockers are still used for those patients with evidence of a vasodilator response at right heart catheter. ${ }^{6}$

An understanding of the molecular pathways involved has resulted in the development of targeted therapies for PAH. There are three main molecules exploited by current therapies: prostacyclin, endothelin- 1 and nitric oxide. ${ }^{2}$ The first therapy developed was the prostacyclin analogue epoprostenol which requires a continuous intravenous infusion via a central tunneled line. The high maintenance requirement and potential complications of this delivery means that it is usually reserved for patients in functional class IV or who have failed to respond to other therapies. ${ }^{6}$
The phosphodiesterase-5 inhibitor sildenafil is licensed for PAH in FC I-IV in the USA and FC II-III in Europe. Endothelin receptor antagonists (ERAs) including bosentan, sitaxentan and ambrisentan are oral therapies licensed for use in PAH. As no oral agent has shown superiority there remains debate as to which should be used first line. A treatment algorithm for patients with PAH is shown in Figure 1 to illustrate the place of ERA therapy. ${ }^{6,10}$

Idiopathic PAH patients make up the majority of study participants, with connective tissue disease associated pulmonary arterial hypertension (CTD-PAH) a significant minority in most trials. Guidance on treatment of specific subtypes is therefore limited and recommendations tend to follow idiopathic PAH guidelines. The use of ERAs is advocated in CTD-PAH as endothelin-1 is felt to play a role in the underlying pathogenesis of these diseases. ${ }^{11}$ Specific studies using bosentan in HIV, congenital heart disease and pediatric patients have been performed. ${ }^{12-14}$ Pulmonary venoocclusive disease and pulmonary capillary hemangiomatosis are rare and pulmonary edema is a significant problem with vasodilator therapy. ${ }^{6}$ Persistent pulmonary hypertension of the newborn is managed by neonatalologists. Patients with chronic thrombo-embolic disease not amenable to surgery can be considered for treatment with specific PAH therapies. ${ }^{15}$ Detailed discussion of the differences between various subgroups and the formulation of treatment algorithms is beyond the scope of this review, and further information can be found in other articles. ${ }^{1,6,16}$

As PAH is a progressive condition it would be an error to consider any form of the disease 'mild'. It is possible with screening to detect it at an early stage, when symptoms may be mild. The easiest way to define such a group is to apply the WHO functional classification and accept those in group II as mildly symptomatic. This is far from perfect and is heavily based on patients' subjective reporting and physicians' interpretation. A more objective definition might use $6 \mathrm{MWD}$ or perhaps the percentage of predicted value, 
possibly with additional Borg dyspnea scores or quality of life measures. There are many potential confounders, eg, chronic adaptation, symptom exacerbation by co-morbid conditions like lung fibrosis and anemia. It is difficult to see how these could be fully accounted for and so any system, however complicated, is likely to remain flawed. Because of the high mortality in untreated cases there are much data on prognostic implications of various parameters, eg, right atrial pressure (RAP), cardiac index (CI), but these do not correlate with symptoms. Therapies are not licensed subject to hemodynamic parameters or 6MWD and consequently functional classification is the major determinant of initial therapy in PAH.

Bosentan has been licensed for the treatment of FC III/IV PAH since 2002 in North America and Europe. At that time treatment was either calcium channel blockers (for those with a positive vasodilator response) or intravenous prostanoids. Bosentan was therefore a practical first-line option for many

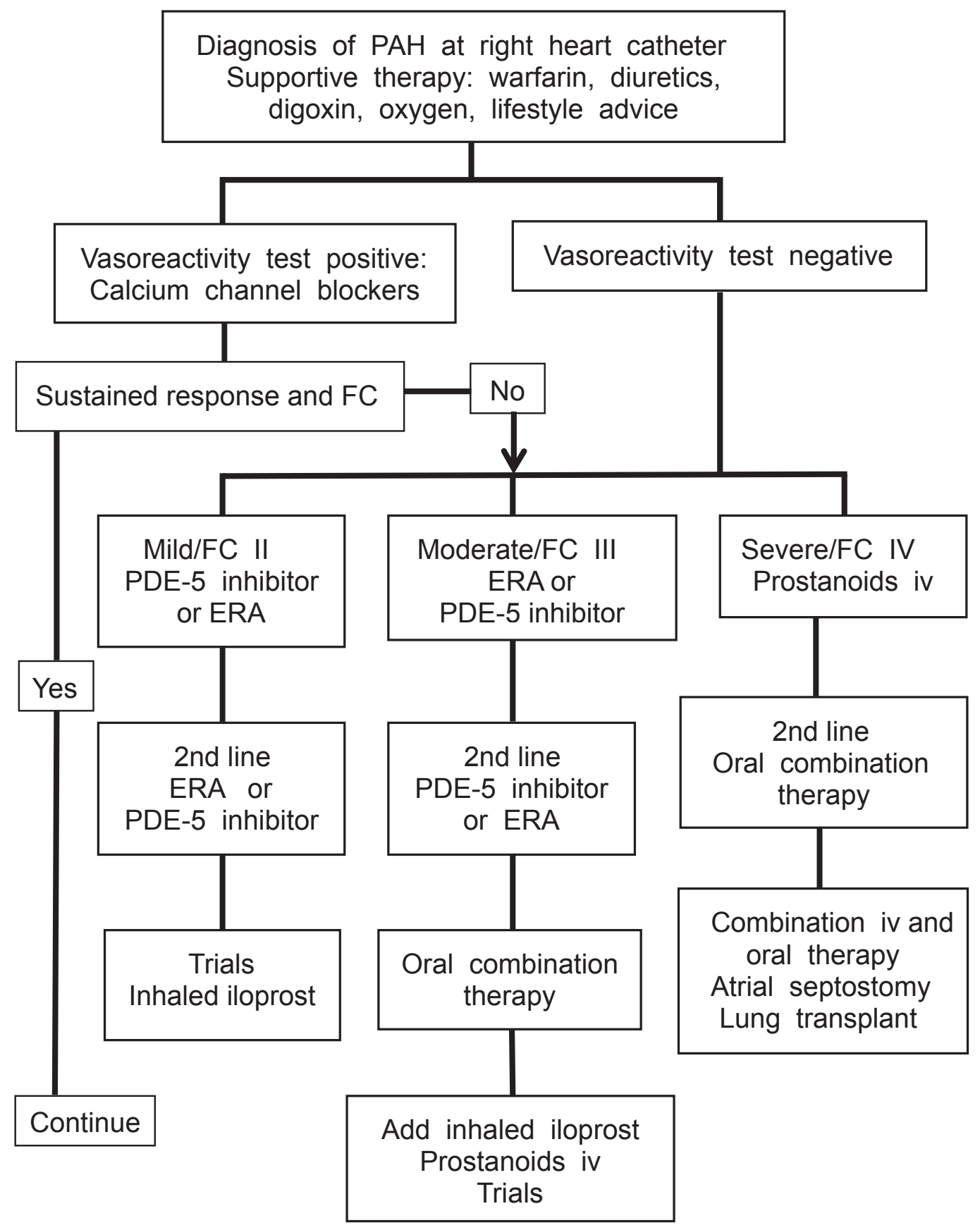

Figure I Outline treatment algorithm for pulmonary arterial hypertension (PAH) at a specialist center. Abbreviations: ERA, endothelin-I receptor antagonist; FC, functional classification. 
patients, and though other oral agents are now available it is still a mainstay of PAH therapy either as monotherapy or, increasingly, as part of combination therapy.

\section{Overview of pharmacology of bosentan in pulmonary arterial hypertension treatment}

The mechanism of action of bosentan is competitive inhibition of endothelin-1 receptors. ${ }^{17}$ Endothelin-1 is a potent vasoconstrictor, which also mediates cell proliferation, fibrosis and inflammation. It is mainly synthesized in endothelial cells and acts locally in autocrine and paracrine fashion. ${ }^{18}$ Two subtypes of endothelin-1 receptor exist; when found on vascular smooth muscle cells they mediate vasoconstriction. Endothelin receptor subtype A $\left(\mathrm{ET}_{\mathrm{A}}\right)$ is predominantly found in smooth muscle and also on fibroblasts, whereas receptor subtype $\mathrm{B}\left(\mathrm{ET}_{\mathrm{B}}\right)$ is expressed on smooth muscle and endothelial cells. ${ }^{19}$ Endothelial ET $_{\mathrm{B}}$ activation mediates clearance of endothelin-1 and vasodilatation by nitric oxide and prostacyclin release. ${ }^{18}$ Because of these effects $\mathrm{ET}_{\mathrm{B}}$ activation is theoretically desirable in PAH. Selective agents with relative $\mathrm{ET}_{\mathrm{A}}: \mathrm{ET}_{\mathrm{B}}$ affinity of greater than 100:1 have been developed, eg, sitaxentan, relative affinity 6500:1, which have also been shown to reduce endothelin-1 levels. ${ }^{19}$ Bosentan exhibits a relative $\mathrm{ET}_{\mathrm{A}}: \mathrm{ET}_{\mathrm{B}}$ affinity of 20:1 by in vitro assays and is therefore classed as a dual ERA. ${ }^{20}$ Although ETA selectivity is attractive, these selective agents have not been shown to be superior to bosentan in clinical trials.

Bosentan is an orally active nonpeptide compound with the chemical formula $\mathrm{C}_{27} \mathrm{H}_{29} \mathrm{~N}_{5} \mathrm{O}_{6} \mathrm{~S}-\mathrm{H}_{2} \mathrm{O} \cdot{ }^{17}$ It is usually started at a dose of $62.5 \mathrm{mg}$ twice daily and uptitrated after 4 weeks to $125 \mathrm{mg}$ twice daily. Bosentan reaches peak plasma concentration three to 5 hours after consumption with an absolute bioavailability of $50 \%$ that is not affected by food. ${ }^{20}$ Bosentan is $98 \%$ bound to albumin with a volume of distribution of $30 \mathrm{~L}$ and a terminal halflife of 5.4 hours. ${ }^{20}$ Steady-state plasma concentrations are achieved in 5 days with a multiple dose regimen. ${ }^{20}$ The pharmacokinetics of bosentan are dose proportional up to $500 \mathrm{mg} /$ day. Adult patients with $\mathrm{PAH}$ have a 2-fold increased exposure compared with pediatric patients and healthy subjects. ${ }^{20}$ Severe renal impairment (creatinine clearance 15 to $30 \mathrm{~mL} / \mathrm{min}$ ) and mild hepatic impairment (Child-Pugh class A) do not have clinically relevant effects, but moderate to severe hepatic impairment are relative contraindications. ${ }^{20}$
Metabolism of bosentan in the liver occurs by the cytochrome p450 enzymes CYP2C9 and CYP3A4, with excretion of metabolites in the bile. One of the three metabolites identified, Ro 48-5033, may be pharmacologically active. ${ }^{20}$ Bosentan is an inducer of the CYP2C9 and CYP3A4 enzymes which may explain the increased clearance and reduced plasma levels of bosentan seen at steady-state. ${ }^{20}$ This induction affects the plasma levels of other compounds metabolized by these enzymes: ciclosporin, glibenclamide, simvastatin and warfarin. Specifically, simvastatin levels may be reduced by $50 \%$; warfarin concentrations are reduced but no relevant changes in INR have been experienced. ${ }^{20}$ Glibenclamide and bosentan concentrations are both reduced when co-administered, and here is also an increased risk of transaminase elevation. ${ }^{20}$ Concomitant ciclosporin use results in a reduced ciclosporin concentration and a tripled bosentan steady-state concentration via CYP3A4. The use of ciclosporin and glibenclamide with bosentan is contraindicated. Inhibitors of CYP2C9 (fluconazole, amiodarone) and CYP3A4 (ketoconazole, itraconazole) can be expected to increase bosentan concentration. ${ }^{20}$ Another important interaction occurs with oral contraceptive agents: concomitant bosentan reduced norethisterone and ethinyl estradiol levels in a pharmacokinetic study and therefore hormonal contraception cannot be relied on. ${ }^{21}$

Bosentan and Ro 48-5033 have been shown to be substrates of the human organic anion transporting polypeptides OATP1B1 and OATP1B3. ${ }^{22}$ Ciclosporin A and rifampicin have been shown to inhibit these hepatic uptake transporter molecules which may explain increases in bosentan levels seen with co-administration. ${ }^{22}$ The increase in bosentan trough levels seen initially with rifampicin turns into a decrease in steady-state conditions probably due to p450 enzyme induction. ${ }^{23}$ Ritonavir also inhibits OATP-mediated uptake and pharmacological studies have shown increased bosentan levels when co-administered with lopinavir and ritonavir. ${ }^{24}$ There was also a small reduction in lopinavir and ritonavir and concomitant use of bosentan and anti-retrovirals requires close monitoring. Both enzyme induction and OATP inhibition may be important in the interaction with sildenafil, another oral therapy used in PAH. In healthy subjects, co-administration of bosentan $125 \mathrm{mg}$ twice daily and sildenafil $80 \mathrm{mg} 3$ times daily resulted in a $50 \%$ rise in bosentan levels and fall in sildenafil by nearly two thirds. ${ }^{25}$ Tadalafil is a phosphodiesterase-5 inhibitor currently in phase III trials for PAH. It is metabolized by CYP3A4 and healthy volunteer studies showed similar results, with reduction in tadalafil levels and increased 
bosentan exposure when co-administered. ${ }^{26}$ This may be clinically important but long-term data are awaited from combination studies in PAH.

\section{Efficacy studies, including comparative studies}

Bosentan has been studied in several clinical PAH trials with generally positive results. The first human study of bosentan treatment used an intravenous preparation which successfully reduced mPAP and PVR. ${ }^{27}$ Unfortunately, it also provoked a drop in systemic arterial pressure and vascular resistance and subsequently an oral preparation has been favored. ${ }^{27}$ After the benefit of oral bosentan in PAH was established in a randomized trial, subsequent papers have attempted to answer several outstanding questions. These include: longterm effect on survival, efficacy in subgroups of PAH and chronic thromboembolic disease, safety and benefit when used in combination with other PAH therapies, and the value in milder disease. A review of the relevant studies with consideration of these questions follows and a summary of bosentan trials is shown in Table $3 .^{12-15,28-36}$

In 2001, a small double-blind, randomized, placebocontrolled trial (study 351) was published. Trial subjects were all in FC III with a 6MWD of 150 to $500 \mathrm{~m}$ and had either idiopathic PAH or PAH associated with scleroderma. After 12 weeks improvement was seen in the primary endpoint, the 6MWD, of $70 \mathrm{~m}$ and $76 \mathrm{~m}$ compared with baseline and placebo respectively. ${ }^{28}$ Repeated hemodynamic studies performed showed marked improvement in mPAP, PVR, RAP and CI compared with placebo as well. ${ }^{28}$ Although all cases started in FC III, by 12 weeks nine of the bosentan treatment arm were in FC II. ${ }^{28}$ The open-label extension showed the effect on 6MWD to be maintained at 6 months and after a year of therapy a significant number $(41.4 \%)$ of patients had improved functional class. ${ }^{37}$ To confirm the positive results of study 351 a multi-center, double-blind, randomized trial was undertaken. BREATHE-1 compared bosentan $125 \mathrm{mg}$ and $250 \mathrm{mg}$ twice daily with placebo over 16 weeks. 213 patients in WHO FC III and IV owing to idiopathic PAH or CTD-PAH with $6 \mathrm{MWD}$ of 150 to $450 \mathrm{~m}$ were enrolled. The observed treatment effect of bosentan on 6MWD was $35 \mathrm{~m}$ for the lower dose, licensed dose. ${ }^{29}$ Other observations made were: improved FC in $42 \%$, now and reduced Borg dyspnea index and prolonged time to clinical worsening (TTCW) compared with placebo. ${ }^{29}$ One-year and 2-year survival data from the bosentan trial patients with idiopathic PAH (96\% and 89\%) compare favorably with National Institutes of Health registry predicted survival rates $\left(69 \%\right.$ and 57\%). ${ }^{38}$ These are not truly

Table 3 PAH studies reporting effect of bosentan

\begin{tabular}{|c|c|c|c|c|c|c|c|c|}
\hline First author & Name & Year & $\mathbf{N}=$ & Groups & Comparator & Design & Effect vs comparator & P-value \\
\hline Channick $^{28}$ & Study 35I & 2001 & 32 & IPAH; SSc & Placebo & DB R & $6 \mathrm{MWD}+76 \mathrm{~m}$ & $\mathrm{P}<0.05$ \\
\hline Rubin $^{29}$ & BREATHE-I & 2002 & 213 & IPAH; CTD & Placebo & DB R & $6 \mathrm{MWD}+35 \mathrm{~m}(250 \mathrm{mg})$ & $\mathrm{P}<0.0 \mathrm{I}$ \\
\hline \multirow[t]{2}{*}{ Barst $^{13}$} & BREATHE-3 & 2003 & 19 & & Baseline & OL & $\mathrm{mPAP}-8 \mathrm{mmHg}$ & \\
\hline & & & & & & & PVR -300 dynes $/ \mathrm{s} / \mathrm{cm}^{5}$ & \\
\hline Humbert ${ }^{30}$ & BREATHE-2 & 2004 & 33 & IPAH; CTD & Placebo & $\mathrm{R}$ & PVR - 188 dynes $/ \mathrm{s} / \mathrm{cm}^{5}$ & ns \\
\hline Sitbon ${ }^{12}$ & BREATHE-4 & 2004 & 16 & HIV & Baseline & OL & $6 M W D+91 \mathrm{~m}$ & $\mathrm{P}<0.00 \mathrm{I}$ \\
\hline Wilkins $^{31}$ & SERAPH & 2005 & 26 & IPAH; CTD & Sildenafil & $\mathrm{R}$ & $6 M W D-16 m$ & ns \\
\hline Barst $^{32}$ & STRIDE-2 & 2006 & 185 & IPAH; CTD; CHD & Sitaxentan & OL R & $6 \mathrm{MWD}+1.5 \mathrm{~m}$ & ns \\
\hline \multirow[t]{2}{*}{ Galiè $^{14}$} & BREATHE-5 & 2006 & 54 & $\mathrm{CHD}$ & Placebo & DB R & PVRI -472 dynes $/ \mathrm{s} / \mathrm{cm}^{5}$ & $\mathrm{P}<0.05$ \\
\hline & & & & & & & $6 M W D+53 \mathrm{~m}$ & $\mathrm{P}<0.0 \mathrm{I}$ \\
\hline Mathai ${ }^{33}$ & & 2006 & 25 & IPAH; SSc & Baseline & $\mathrm{OL}$ & $6 M W D+52 m$ & ns \\
\hline Denton $^{34}$ & TRUST & 2007 & 53 & CTD & Baseline & $\mathrm{OL}$ & I 2 patients improved FC & \\
\hline Akagi $^{35}$ & & 2008 & 8 & IPAH & Baseline & $\mathrm{OL}$ & $\mathrm{mPAP}-13 \mathrm{mmHg}$ & $\mathrm{P}<0.05$ \\
\hline \multirow[t]{2}{*}{ Galiè $^{36}$} & EARLY & 2008 & 185 & IPAH; CHD; CTD; HIV & Placebo & DB R & $6 M W D+19 m$ & ns \\
\hline & & & & & & & PVR - 197 dynes $/ \mathrm{s} / \mathrm{cm}^{5}$ & $\mathrm{P}<0.0001$ \\
\hline \multirow[t]{2}{*}{$J_{a i i s}{ }^{15}$} & BENEFIT & 2008 & 99 & CTEPH & Placebo & DB R & PVR - 193 dynes $/ \mathrm{s} / \mathrm{cm}^{5}$ & $\mathrm{p}<0.0001$ \\
\hline & & & & & & & $6 M W D+2 m$ & ns \\
\hline
\end{tabular}

Abbreviations: IPAH, idiopathic pulmonary artery hypertension; SSc, scleroderma-associated pulmonary arterial hypertension; CTD, connective tissue disease associated pulmonary artery hypertension; HIV, human immunodeficiency virus associated pulmonary artery hypertension; CHD, congenital heart disease associated pulmonary artery hypertension; CTEPH, chronic thromboembolic pulmonary hypertension; DB, double blinded; R, randomized; OL, open label, 6MWD, six-minute walk distance; mPAP, mean pulmonary artery pressure; PVR, pulmonary vascular resistance; FC, functional class. 
comparable data, but are important because trials in PAH have been unable to show survival benefit in the short controlled phases (a frequent criticism).

The majority of study participants in BREATHE-1 were idiopathic PAH patients. Subgroup analysis of BREATHE-1 by diagnosis suggests a difference in response. Bosentan improved 6WMD in idiopathic PAH, whereas it prevented deterioration in CTD-PAH: a $3 \mathrm{~m}$ improvement was seen compared to a $40 \mathrm{~m}$ decline in placebo-treated CTD-PAH patients. ${ }^{29}$ Combined analysis of the CTD-PAH patients in study 351 and BREATHE-1 suggests a trend favoring bosentan with a $22 \mathrm{~m}$ improvement in 6MWD. ${ }^{39}$ This was despite less favorable baseline 6MWD and PVR. Survival estimates of $86 \%$ and $73 \%$ at 1 and 2 years, respectively, although better than historical data, were not as good as for idiopathic PAH. ${ }^{39}$ The TRUST study sought to assess survival and quality of life in CTD-PAH patients. At 48 weeks, the survival was $92 \%$ with absence of clinical worsening in $68 \%$ by Kaplan-Meier estimation. ${ }^{34}$ The broader implication of these data is that benefit seen in one type of PAH may not be manifest in another. The BREATHE-4 study demonstrated benefit in 16 patients with HIV associated PAH. Sixteen weeks of bosentan therapy improved 6MWD to $424 \mathrm{~m}$ from $333 \mathrm{~m}$ at baseline, with hemodynamic improvement and 13 patients attaining FC II status. ${ }^{12}$ A placebo-controlled study (BREATHE-5) looked at the effects of bosentan in 52 subjects with Eisenmenger syndrome with an added safety endpoint of systemic oxygen saturation. 6MWD, mPAP and PVR were significantly improved with only a $1 \%$ drop in oxygen saturations. ${ }^{14}$ Data from the open-label extension reported an overall improvement in 6MWD at 24 weeks with a drop of only $0.5 \%$ in systemic arterial saturations. ${ }^{40} \mathrm{~A}$ multi-center trial of bosentan in patients with inoperable chronic thromboembolic pulmonary hypertension showed hemodynamic improvement but not benefit in exercise capacity. ${ }^{15}$

As PAH is a progressive condition it seems reasonable to suppose that early treatment can prevent clinical deterioration in mildly symptomatic patients. Most trials have reported subjects improving from FC III to II before the open-label extension. Therefore many patients in FC II have received treatment, albeit they had to deteriorate to FC III status first. Unfortunately this reflects what happens to many FC II patients in practice. The combined open-label extension studies do not contain enough evidence to justify treating those in FC II. Although patients in FC II have higher mortality and event rates than healthy subjects, these rates are lower than seen in FC III and IV patients. An event-driven outcome would therefore require a longer placebo-controlled period than was ethically acceptable for the early PAH trials.
BREATHE-1 reported a diminished 6MWD treatment effect in patients with more favorable hemodynamics: mPAP of $<50 \mathrm{mmHg}$ and $\mathrm{CI} \geq 2.3 \mathrm{~L} / \mathrm{min} / \mathrm{m}^{2} .{ }^{29}$ The limited effect on 6MWD with sitaxentan in STRIDE-1 raised concerns of a ceiling effect in 6MWD improvement, as no upper limit of 6MWD was specified. ${ }^{41}$ Trials with disappointing results may point to the inclusion of those with milder PAH as a potential confounder, but several trials with a minority of FC II patients have posted significant results (see Table 4). ${ }^{32,36,42-50}$ Enough FC I and II patients were treated in the SUPER-1 and ARIES trials to show effect on 6MWD in these subgroups. ${ }^{42,44}$ Long-term follow-up data from patients treated with the ERA ambrisentan show improved 6MWD at 2 years, relative to baseline, and estimated survival of $95 \% .^{51}$

The EARLY trial remains the only PAH trial designed specifically for FC II patients. It was prospective, doubleblinded, randomized and placebo-controlled. Patients with idiopathic, familial, HIV, anorexigen, connective tissue disease and congenital heart disease (with some restrictions) associated PAH were recruited. ${ }^{36}$ Rather than fixing an absolute upper limit for the baseline 6MWD a value of less than $80 \%$ predicted or $500 \mathrm{~m}$ with a Borg score $\geq 2$ and a PVR $\geq 320$ dynes $/ \mathrm{s} / \mathrm{cm}^{5}$ were specified. Co-primary endpoints of change in PVR and 6MWD at 6 months were used. Secondary endpoints included TTCW and change from baseline to 6 months in: WHO FC, Borg score, RAP, mPAP, CI and mixed venous oxygen saturation $\left(\mathrm{mVO}_{2}\right)$. Definitions of TTCW vary between trials. EARLY used death, hospitalization due to PAH complications and symptomatic progression (new or worsening right heart failure or a $10 \%$ reduction in $6 \mathrm{MWD}$ from baseline assessed twice more than 2 weeks apart or 5\% reduction in 6MWD from baseline assessed more than 2 weeks apart with an increase in Borg score of at least 2 points). Using this definition the clinical worsening event rate was $14 \%$ in the placebo group over 6 months. ${ }^{36}$

Of the 476 patients screened 139 were not felt to be in functional class II and 76 met exclusion criteria. ${ }^{36}$ Due to trial rules only 81 patients taking bosentan and 82 assigned placebo completed the study. Mean 6MWD data suggested a trend towards improvement with bosentan: $+11.2 \mathrm{~m}$ versus $-7.9 \mathrm{~m}$ for placebo. ${ }^{36}$ Analysis of PVR values using the Wilcoxon rank-sum test showed a significant decrease in PVR for the bosentan group $(83.2 \%$ versus placebo $107.5 \%) .{ }^{36}$ Other hemodynamic variables that showed improvement were mPAP, $\mathrm{CI}$ and $\mathrm{mVO}_{2} \cdot{ }^{36} \mathrm{TTCW}$ was delayed with bosentan therapy $(\mathrm{p}<0.02)$ compared with placebo with a hazard ratio of $0.23 .{ }^{36}$ Twelve patients taking 
Table 4 PAH studies including (more than 10) patients in FC II

\begin{tabular}{|c|c|c|c|c|c|c|c|c|}
\hline Author/study & Year & Treatment & Design & Endpoint & Effect & No. FC II & Treat & $\begin{array}{l}\text { FC II } \\
\text { analysis }\end{array}$ \\
\hline Galiè SUPER-I ${ }^{42}$ & 2005 & sildenafil & DB R P & 6MWD & $+42 \mathrm{~m} \mathrm{p}<0.001$ & 108 of 278 & $75^{*}$ & $+40 \mathrm{~m}$ \\
\hline Galiè $^{43}$ & 2005 & ambrisentan & DB R P & 6MWD & $+36 \mathrm{~m} \mathrm{p}<0.0001$ & 23 of 64 & 17 & $+58 \mathrm{~m}$ \\
\hline \multirow[t]{2}{*}{ Galiè EARLY ${ }^{36}$} & 2008 & bosentan & DB R P & PVR & $-197 p<0.0001$ & 185 of 185 & 93 & $\mathrm{n} / \mathrm{a}$ \\
\hline & & & & 6MWD & $+19 \mathrm{~m} \mathrm{~ns}$ & & & $\mathrm{n} / \mathrm{a}$ \\
\hline Galiè ARIES ${ }^{44}$ & 2008 & ambrisentan & DB R P & 6MWD & $+43 \mathrm{~m}$ & 165 of 383 & $113^{*}$ & $36-55 m$ \\
\hline Simonneau UTI $5^{45}$ & 2002 & trepostinil & DB R P & 6MWD & $+10 \mathrm{~m}$ & 53 of 470 & 25 & No \\
\hline Galiè ALPHABET ${ }^{46}$ & 2002 & beraprost & DB R P & 6MWD & $+15 \mathrm{~m}$ & 64 of 130 & 31 & No \\
\hline Barst $^{47}$ & 2003 & beraprost & DB R P & $\mathrm{VO}_{2} \max$ & $\mathrm{P}<0.002$ & 61 of 116 & 33 & No \\
\hline Barst STRIDE-I ${ }^{48}$ & 2003 & sitaxentan & DB R P & $\mathrm{VO}_{2} \max$ & $+\mathrm{I} . \mathrm{I}$ & 59 of 178 & 37 & No \\
\hline Sastry ${ }^{49}$ & 2004 & sildenafil & $D B \times P$ & Treadmill test & $+2 \mathrm{IIs} \mathrm{p}<0.000 \mathrm{I}$ & 18 of 22 & 18 & No \\
\hline \multirow[t]{2}{*}{ Barst STRIDE-2 $2^{32}$} & 2006 & sitaxentan & DB R P & 6MWD & $+28 \mathrm{~m} \mathrm{p}<0.05$ & 92 of 185 & 47 & No \\
\hline & & bosentan & & 6MWD & $+29 \mathrm{~m} \mathrm{p}<0.05$ & & 22 & No \\
\hline Simonneau PACES ${ }^{50}$ & 2008 & sildenafil & DB R P & 6MWD & $+29 \mathrm{~m}$ & 71 of 267 & $35 *$ & No \\
\hline
\end{tabular}

Notes: Treat column refers to the number of patients enrolled in each trial in FC I or II and assigned to treatment arm. *includes FC I patients.

Abbreviations: DB, double blind; R, randomized; P, placebo-controlled; $X$, crossover; 6MWD, six-minute walk distance; PVR, pulmonary vascular resistance; FC, functional class.

placebo progressed to FC III compared with only 3 patients on bosentan $(\mathrm{p}<0.05)$. N-terminal-pro-BNP levels were only assessed in certain pre-specified centers; the treatment effect was $-471 \mathrm{ng} / \mathrm{L}(\mathrm{p}<0.0005)$ comparing bosentan with placebo. ${ }^{36}$ Overall the results were therefore positive, even if some of the selection criteria were unusual.

With specific therapies for PAH acting on three different pathways and their efficacy as monotherapy proven, a logical step forward has been to look at combining agents. In BREATHE-2, 33 patients starting intravenous epoprostenol were randomized to either additional placebo or bosentan. Assessment at 16 weeks showed 6MWD and functional class improvement in both groups with a trend towards better hemodynamics in the bosentan-treated patients. ${ }^{30} \mathrm{~A}$ small study conducted in Japan also showed improvement with the addition of bosentan to epoprostenol in idiopathic PAH. Eight patients on stable doses of epoprostenol (average $100 \mathrm{ng} / \mathrm{kg} / \mathrm{min}$ ) had continuous hemodynamic monitoring for 2 days during initiation with $62.5 \mathrm{mg}$ bosentan twice daily. ${ }^{35}$ Even while reducing epoprostenol to maintain $\mathrm{SvO} 2$ at baseline value, systolic pulmonary artery pressure and PVR fell; these changes were maintained in six of the patients at 1 year. ${ }^{35}$

Several investigators have looked at the combination of bosentan and sildenafil. In an observational cohort of 9 patients with idiopathic PAH adjunctive therapy was deemed necessary after several months due to deterioration. ${ }^{52}$ Initial response to bosentan was significant $(+57 \mathrm{~m})$ to an average of $403 \mathrm{~m}$, but this fell to just $277 \mathrm{~m}$ at the time of adding sildenafil between 6 and 16 months later. The resulting increase in 6MWD $(+122 \mathrm{~m})$ was sustained for 6 months. ${ }^{52}$ In a group including 13 idiopathic PAH and twelve scleroderma-associated PAH patients started on bosentan monotherapy, sildenafil was offered instead of intravenous therapy, after clinical deterioration. Improvement in FC and 6MWD was only seen in the idiopathic PAH group however. ${ }^{33}$ Sildenafil received a license for use in PAH while the EARLY trial was ongoing. Because of this development an amendment was made to the study protocol allowing concomitant sildenafil use; provided it was started more than one month before enrolment and the dose remained steady during the trial. The FC status of these 28 patients before commencing sildenafil is not known. The reduction in PVR in this group was similar to that seen in the main analysis but the confidence interval ranged from $-44 \%$ to $13 \%$ because of the small numbers. ${ }^{36} \mathrm{~A}$ trend towards reduction in 6MWD was also seen in this subgroup. ${ }^{36}$

The effects of bosentan have also been compared with other specific therapies for PAH. The SERAPH investigators randomized 26 patients with PAH in FC III to either standard regimen bosentan or sildenafil ( $50 \mathrm{mg}$ twice daily for 4 weeks, then $50 \mathrm{mg} 3$ times daily). ${ }^{31}$ At 16 weeks there were no significant differences in effect between the two drugs: bosentan improved 6MWD by $59 \mathrm{~m}$ compared with $75 \mathrm{~m}$ in the sildenafil group. ${ }^{31}$ Both therapies improved CI, but only sildenafil reduced plasma BNP levels ${ }^{31}$ A survival comparison with a historical cohort of 346 IPAH patients treated with epoprostenol favored bosentan. One hundred 
and thirty-nine patients in FC III at commencement of bosentan had a survival of $97 \%$ and $91 \%$ at 1 and 2 years compared with $91 \%$ and $84 \%$ with epoprostenol. ${ }^{53}$ The STRIDE-2 trial included an open-label bosentan arm in addition to the placebo and sitaxentan treatment arms. After 18 weeks, sitaxentan $100 \mathrm{mg}$ and bosentan both increased 6MWD; the difference in treatment effect was less than two metres. ${ }^{32}$ Sitaxentan also improved functional class but neither treatment delayed time to clinical worsening. ${ }^{32}$ The extension study converted all patients to $100 \mathrm{mg}$ dose and followed up for 1 year. No difference was seen in 6MWD or change in functional class. ${ }^{54}$ For the CTD-PAH subgroup; the difference in TTCW reached significance favoring sitaxentan (hazard ratio 0.20). ${ }^{55}$ There are no data comparing bosentan and ambrisentan but efficacy data from trials with the three available ERAs do not suggest superiority of any one agent.

\section{Safety and tolerability}

The ERA class of drugs are associated with many side effects and even though significant safety problems are rare, there are many cautions necessary for their use. As discussed earlier, the pharmacology of bosentan and ERAs can induce hepatic enzymes making hormonal contraception unreliable. Of course they are contraindicated in pregnancy as well due to teratogenicity. Breast-feeding is not recommended either because it is unknown whether ERAs pass into breast milk. The safety of bosentan use in children with PAH has been explored in an open label study which confirmed safe dose reduction in the pediatric population. Concurrent use of ciclosporin A with any ERA is contraindicated and glibenclamide with bosentan due to drug-drug interactions. Experience with bosentan in HIV associated PAH is limited and interaction with anti-retroviral drugs requires careful monitoring for the same reasons. ${ }^{12}$ Clinical studies report very few patients stopping bosentan, but many experiencing adverse effects.

The principal problem with bosentan is hepatotoxicity initially manifest as elevated levels of alanine aminotransferase and aspartate aminotransferase. The bosentan information insert quotes an incidence of $11 \%$ for elevation of these liver enzymes to levels more than 3 times the upper limit of normal (ULN). ${ }^{56}$ These changes are dosedependent; in BREATHE-1 there was a 14\% incidence of hepatic enzyme elevation in the $250 \mathrm{mg}$ twice daily group compared with $4 \%$ in the $125 \mathrm{mg}$ arm and $3 \%$ in the placebo arm. ${ }^{29}$ Levels above 8 times the ULN occurred in 2 patients on the $125 \mathrm{mg}$ twice daily and 5 patients taking
$250 \mathrm{mg}$ twice daily. ${ }^{29}$ In BREATHE-5, 2 of the 37 patients developed elevated transaminases; this led to discontinuation in one case. ${ }^{14}$ In TRUST, $17 \%$ of CTD-PAH patients developed elevated liver enzymes, requiring discontinuation of bosentan in three. ${ }^{34}$ Postmarketing surveillance reports led to a strengthening of the warning on monthly liver enzyme monitoring. This includes one case of biopsy confirmed cirrhosis in a patient taking bosentan. ${ }^{57}$ Although the patient was suffering from an intravenous catheter related infection and subsequently recovered; a contribution of bosentan to the liver damage could not be excluded. ${ }^{57}$ A more recent case of hepatotoxicity that developed with the addition of methotrexate, resolved after bosentan was stopped and did not recur with reintroduction of methotrexate. ${ }^{58}$ Currently, product guidance recommends stopping bosentan if levels are more than 5 times ULN, and re-introduction can only be considered if levels were less than 8 times ULN. For levels between 3 and 5 times ULN dose reduction may be adequate, but fortnightly monitoring is recommended. ${ }^{56}$ Although lasting damage has occurred rarely, hepatic enzyme elevation is the most frequently cited reason for bosentan discontinuation. The reported incidence of elevated transaminases is lower with sitaxentan $(7 \%)$ and ambrisentan $(<1 \%)$ therapy. It should be noted that the incidence in the placebo groups in these trials is also lower. ${ }^{19}$

Hemoglobin levels fall by approximately $0.9 \mathrm{~g} / \mathrm{dL}$ with bosentan therapy, predominantly in the first few weeks of therapy with stabilization beyond 12 weeks. ${ }^{20}$ A decrease of $>1 \mathrm{~g} / \mathrm{dL}$ was seen in $68 \%$ of patients treated with bosentan compared with $29 \%$ on placebo. ${ }^{56}$ Three patients in study 351 had significant falls in hemoglobin recorded; levels did not drop below $10.4 \mathrm{~g} / \mathrm{dL}$ though. ${ }^{28}$ One patient in BREATHE-5 developed a significant anemia with a hemoglobin of $<10 \mathrm{~g} / \mathrm{dL}$. ${ }^{14,40}$ The effect of chronic oral therapy on blood pressure does not seem to be dramatic; in contrast to the intravenous preparation. Mean systemic arterial pressure fell by $3 \mathrm{mmHg}$ in BREATHE- 1 and $4 \mathrm{mmHg}$ in BREATHE-5. ${ }^{14,29}$ A safety endpoint was included in BREATHE-5 because of concerns that saturations may be reduced by bosentan. There was a nonsignificant, small reduction in mixed venous saturations in the bosentan group with no adverse events reported as a result. ${ }^{14}$

When bosentan was trialled as therapy for chronic heart failure it provoked increased peripheral edema within weeks. ${ }^{18}$ This effect has been documented in PAH with an incidence of $10 \%$ in study 351 and many patients anecdotally requiring diuretics or hospital treatment. ${ }^{28}$ 
Rates in other PAH trials are higher, eg, BREATHE-2 27\%, BREATHE-5 19\% and TRUST 17\%. ${ }^{14,30,34}$ The mechanism of edema is not entirely clear; in some patients it may be sequelae of worsening right heart failure (and some trials have considered it so). It may be the result of vasodilatation caused by ERAs, or from effects upon renal tubular function. ${ }^{18}$ In the open-label extension of study 351, a large number of adverse symptoms were reported: headache, dizziness, cough, dyspnea, syncope, flushing, respiratory tract infection, chest pain, palpitation, fatigue, arthralgia, dyspepsia, epistaxis and nausea. ${ }^{28}$ Many of these might be attributable to progression of PAH and were not seen with significantly greater frequency in the bosentan groups compared with placebo in BREATHE-1. ${ }^{29}$ Headache, dizziness, palpitations and chest pain were seen more frequently in the bosentan group in BREATHE-5. ${ }^{14}$ Diarrhea, exacerbated dyspnea and nausea were reported in more than $10 \%$ of TRUST participants. ${ }^{34}$ These differences in reported side effects may reflect the different study populations.

From the limited amount of data available, most of which is not placebo-controlled, it seems combination therapy is well tolerated. In one report of bosentan and sildenafil combination no greater number of liver enzyme elevations were seen compared with bosentan monotherapy. ${ }^{10}$ In another trial several patients had to stop sildenafil for severe dyspepsia (mostly CTD-PAH). Two patients developed LFT abnormalities after addition of sildenafil - one resolved with discontinuation of sildenafil, the other with reduction of bosentan. One patient developed intractable headaches which required discontinuation of sildenafil. In BREATHE-2 peripheral edema was significantly more common in the bosentan/epoprostenol group, although liver enzyme elevation was seen more often in the placebo/epoprostenol group. ${ }^{30}$

Obviously, when using a drug in situations where there may be less positive clinical effect, safety must come under closer scrutiny to ensure that the risk-benefit ratio is still favorable. The commonest adverse events in the bosentan group in the EARLY trial were nasopharyngitis and elevated liver enzymes. ${ }^{36}$ Twelve patients on bosentan developed transaminase levels over 3 times ULN, 10 within 20 weeks. In all cases transaminase levels returned towards baseline levels with appropriate management (at least 6 discontinued altogether). In the open-label extension seven serious adverse events occurred: cardiac failure and anemia, progression of PAH, abnormal liver enzymes $(n=3), 1$ death after seizures probably caused by vasculitis reactivation, and an episode of toxic epidermal necrolysis and acute hepatitis. ${ }^{36}$ Postmarketing surveillance reports have flagged hypersensitivity, rash, thrombocytopenia, jaundice, anemia requiring transfusion and hepatic cirrhosis/failure as serious adverse events. These potentially grave consequences bear thought when considering therapy in those with mild symptoms.

\section{Patient-focused outcomes such as quality of life}

Several different scores can be used to quantify the patient's perception of improvement in PAH. The Borg dyspnea index or score is the most widely used patient-reported measure in PAH trials; a fall in the score suggests less exertional breathlessness. This was seen in study 351 and BREATHE-1 with a net treatment effect of -0.6 (CI -1.2 to -0.1$)$ in the latter. ${ }^{28,29}$ BREATHE-4 found bosentan improved Borg score in HIV associated PAH (1.5 vs 3.4 at baseline, $\mathrm{p}<0.02) .{ }^{12}$ Perhaps it should have been expected that there was no change in Borg score was seen in the EARLY trial. ${ }^{36}$ BREATHE-2 used a dyspnea-fatigue index which did not change with the addition of bosentan. ${ }^{30}$

PAH trials have not consistently used any one symptom scoring system. Although the camphor score was specifically designed for PAH the different aims of trials means it is often not suited for this purpose. Quality of life assessed by the EQ-5D visual analog scale improved in BREATHE-4 ( $p<0.001)$, as did 6 of the 8 items in the SF-36 health survey form: physical functioning, role-physical, general health perception, vitality, social functioning, mental health. ${ }^{12}$ The TRUST study analyzed a broader set of items looking for additional benefits in connective tissue disease patients. There were minimal improvements at 48 weeks in SF-36 scores; more patients felt they had improved than deteriorated on the health transition item (mean -0.83 , 95\% confidence intervals -1.27 to -0.39$).{ }^{34}$ The health assessment questionnaire and visual analogue scores used tended to increase suggesting a lack of perceived benefit. ${ }^{34}$ In EARLY the SF-36 health questionnaire scores suggested that $57 \%$ of bosentan patients and $38 \%$ of placebo patients felt their condition had improved $(\mathrm{p}<0.05) \cdot{ }^{36}$ Breakdown of domains did not show significance for any individual part, the most marked trend was for general health perceptions followed by role-physical and mental health and vitality.

\section{Conclusions, place in therapy}

The progressive natural history of PAH gives us a clear rationale for treating the disease early. However, the 
criteria we use to decide who to treat and how we treat them are still open to debate. The combined complexities of ethics and study design for this patient group mean the optimal trial endpoints are not clear. The EARLY trial shows that unless they are treated we can expect patients in FC II to progress to a more symptomatic state. Recent meta-analysis of PAH trials has demonstrated improved survival with targeted therapy; resulting in a number needed to treat of 20 to prevent one death at 1 year. ${ }^{59}$ While survival is perhaps the dominant concern in patients with more severe disease, ideally those at the milder end of the spectrum would not progress that far. Those therapies which provide mortality benefit in severe disease may not delay progression in the earlier stages as effectively as other agents. Advocates of targets in $\mathrm{PAH}$ therapy would include FC II status as a clear goal. Thus, many PAH patients who are in FC II are treated, if only because they have shown clinical evidence of deterioration previously. Waiting for such evidence before commencing therapy is not a logical approach. The EARLY trial provides clear evidence for treating the FC II patient; this is supported by sub-group data from other trials, eg, ARIES. ${ }^{36,44}$

Bosentan has been shown to be effective at improving exercise tolerance in PAH patients in FC III and IV in randomized controlled trials. Of the currently available oral therapies for PAH there is more long-term data relating to bosentan use. The existence of small studies which have addressed specific problems and subgroups provides reassurance when confronted with the more unusual cases. In the absence of comparative data showing superiority of any oral therapy in terms of clinical effect, prescribing decisions may be based on other factors. Given the likelihood that a treatment for FC II patients may be needed for several years the ideal drug would be one without significant side effects or drug interactions. No oral therapy is free of adverse effects but bosentan has perhaps a worse side effect profile than the other ERAs and phosphodiesterase-5 inhibitors. Longterm and post-marketing data are awaited to see if the lower incidence of adverse events reported in trials of other agents persists. In conclusion, bosentan seems to offer benefit to those patients with PAH in FC II, but the relatively high incidence of adverse events may be prohibitive for some patients and clinicians.

\section{Disclosure}

CJ Valerio had no conflicts of interest to declare. JG Coghlan has received consultancy and lecture fees from Actelion,
GlaxoSmithKline, Pfizer and Gilead. His department has benefited from research grants from Actelion and Pfizer.

\section{References}

1. Simonneau G, Galiè N, Rubin LJ, et al. Clinical classification of pulmonary hypertension. J Am Coll Cardiol. 2004;43 Suppl S: $5 \mathrm{~S}-12 \mathrm{~S}$.

2. Farber HW, Loscalzo J. Mechanisms of disease: pulmonary hypertension. New Engl J Med. 2004;351:1655-1665.

3. Barst R, McGoon M, Torbicki A, et al. Diagnosis and differential assessment of pulmonary arterial hypertension. $J$ Am Coll Cardiol. 2004;43 Suppl:S40-S47.

4. Sun XG, Hansen JE, Oudiz RJ, et al. Exercise pathophysiology in patients with primary pulmonary hypertension. Circulation. 2001;104:429-435.

5. McGoon M, Gutterman D, Steen V, et al. Screening, early detection, and diagnosis of pulmonary arterial hypertension: ACCP Evidence-Based Clinical Practice Guidelines. Chest. 2004;126 Suppl;S14-S34.

6. Galiè N, Torbicki A, Barst R, et al. Guidelines on diagnosis and treatment of pulmonary arterial hypertension. The Task Force on Diagnosis and Treatment of Pulmonary Arterial Hypertension of the European Society of Cardiology. Eur Heart J. 2004;25:2243-2278.

7. Miyamoto S, Nagaya N, Satoh T, et al. Clinical correlates and prognostic significance of six-minute walk test in patients with primary pulmonary hypertension: comparison with cardiopulmonary exercise testing. Am J Resp Crit Care Med. 2000;161: 487-492.

8. Sanchez O, Sitbon O, Jaïs X, Simonneau G, Humbert G. Immunosuppressive therapy in connective tissue diseases-associated pulmonary arterial hypertension. Chest. 2006;130:182-189.

9. Jais X, Launay D, Yaici A, et al. Immunosuppressive therapy in lupusand mixed connective tissue disease-associated pulmonary arterial hypertension a retrospective analysis of twenty-three cases. Arthritis Rheum. 2008;58:521-531.

10. Hoeper MM, Markevych I, Spiekerkoetter E, Welte T, Niedermeyer J. Goal-oriented treatment and combination therapy for pulmonary arterial hypertension. Eur Respir J. 2005;26:858-863.

11. Gibbs S, Corris P, eds; for National pulmonary hypertension centres of the UK and Ireland. Consensus statement on the management of pulmonary hypertension in clinical practice in the UK and Ireland. Heart. 2008;94 Suppl I:i1-i41.

12. Sitbon O, Gressin V, Speich R, et al. Bosentan for the treatment of human immunodeficiency virus-associated pulmonary arterial hypertension. Am J Respir Crit Care Med. 2004;170:1212-1217.

13. Barst RJ, Ivy D, Dingemanse J, et al. Pharmacokinetics, safety, and efficacy of bosentan in pediatric patients with pulmonary arterial hypertension. Clin Pharmacol Ther. 2003;73:372-382.

14. Galiè N, Beghetti M, Gatzoulis MA, et al. Bosentan therapy in patients with Eisenmenger syndrome: a multicenter, double-blind, randomized, placebo-controlled study. Circulation. 2006;114:48-54.

15. Jaïs X, D’Armini AM, Jansa P, et al. Bosentan for treatment of inoperable chronic thromboembolic pulmonary hypertension: BENEFiT (Bosentan Effects in iNopErable Forms of chronic Thromboembolic pulmonary hypertension), a randomized, placebo-controlled trial. $J \mathrm{Am}$ Coll Cardiol. 2008;52:2127-2134.

16. McLaughlin VV, Archer SL, Badesch DB, et al. ACCF/AHA 2009 Expert Consensus Document on Pulmonary Hypertension: A Report of the American College of Cardiology Foundation Task Force on Expert Consensus Documents and the American Heart Association. J Am Coll Cardiol. 2009;53:1573-1619.

17. Kenyon KW, Nappi JM. Bosentan for the treatment of pulmonary arterial hypertension. Ann Pharmacother. 2003;37:1055-1062.

18. Motte S, McEntee K, Naeije R. Endothelin receptor antagonists. Pharmacol Ther. 2006;110:386-414. 
19. Opitz CF, Ewert R, Kirch W, Pittrow D. Inhibition of endothelin receptors in the treatment of pulmonary arterial hypertension: does selectivity matter? Eur Heart J. 2008;29:1936-1948.

20. Dingemanse J, van Giersbergen PL. Clinical pharmacology of bosentan, a dual endothelin receptor antagonist. Clin Pharmacokinet. 2004;43:1089-1115.

21. van Giersbergen PL, Halabi A, Dingemanse J. Pharmacokinetic interaction between bosentan and the oral contraceptives norethisterone and ethinyl estradiol. Int J Clin Pharmacol Ther. 2006;44: 113-118.

22. Treiber A, Schneiter R, Häusler S, Stieger B. Bosentan is a substrate of human OATP1B1 and OATP1B3: inhibition of hepatic uptake as the common mechanism of its interactions with cyclosporin A, rifampicin, and sildenafil. Drug Metab Dispos. 2007;35:1400-1407.

23. van Giersbergen PL, Treiber A, Schneiter R, Dietrich H, Dingemanse J. Inhibitory and inductive effects of rifampin on the pharmacokinetics of bosentan in healthy subjects. Clin Pharmacol Ther. 2007;81: 414-419.

24. McRae MP, Lowe CM, Tian X, et al. Ritonavir, saquinavir, and efavirenz, but not nevirapine, inhibit bile acid transport in human and rat hepatocytes. J Pharmacol Exp Ther. 2006;318:1068-1075.

25. Burgess G, Hoogkamer H, Collings L, Dingemanse J. Mutual pharmacokinetic interactions between steady-state bosentan and sildenafil. Eur J Clin Pharmacol. 2008;64:43-50.

26. Wrishko RE, Dingemanse J, Yu A, Darstein C, Phillips DL, Mitchell MI. Pharmacokinetic interaction between tadalafil and bosentan in healthy male subjects. J Clin Pharmacol. 2008;48:610-618.

27. Williamson DJ, Wallman LL, Jones R, et al. Hemodynamic effects of bosentan, an endothelin receptor antagonist, in patients with pulmonary hypertension. Circulation. 2000;102:411-418.

28. Channick RN, Simmoneau G, Sitbon O, et al. Effects of the dual endothelin-receptor antagonist Bosentan in patients with pulmonary hypertension: a randomised placebo-controlled study. Lancet. 2001;358:1119-1123.

29. Rubin LJ, Badesch DB, Barst RJ, et al. Bosentan therapy for pulmonary arterial hypertension. New Eng J Med. 2002;346:896-903.

30. Humbert M, Barst RJ, Robbins IM, et al. Combination of bosentan with epoprostenol in pulmonary arterial hypertension: BREATHE-2. Eur Respir J. 2004;24:353-359.

31. Wilkins MR, Paul GA, Strange JW, et al. Sildenafil versus Endothelin receptor Antagonist for Pulmonary Hypertension (SERAPH) study. Am J Respir Crit Care Med. 2005;171:1292-1297.

32. Barst RJ, Langleben D, Badesch D, et al; for STRIDE-2 Study Group. Treatment of pulmonary arterial hypertension with the selective endothelin-A receptor antagonist sitaxsentan. J Am Coll Cardiol. 2006;47:2049-2056.

33. Mathai SC, Girgis RE, Fisher MR, et al. Addition of sildenafil to bosentan monotherapy in pulmonary arterial hypertension. Eur Respir J. 2007;29:469-475.

34. Denton CP, Pope JE, Peter HH, et al. Long-term effects of bosentan on quality of life, survival, safety and tolerability in pulmonary arterial hypertension related to connective tissue diseases. Ann Rheum Dis. 2008;67:1222-1228.

35. Akagi S, Matsubara H, Miyaji K, et al. Additional effects of bosentan in patients with idiopathic pulmonary arterial hypertension already treated with high-dose epoprostenol. Circ J. 2008;72:1142-1146.

36. Galiè N, Rubin LJ, Hoeper MM, et al. Treatment of patients with mildly symptomatic pulmonary arterial hypertension with bosentan (EARLY study): a double-blind, randomised controlled trial. Lancet. 2008;371:2093-2100.

37. Sitbon O, Badesch D, Channick RN, et al. Effects of the dual endothelin receptor antagonist bosentan in patients with pulmonary arterial hypertension. A 1-year follow-up study. Chest. 2003; 124:247-254.

38. McLaughlin VV. Survival in patients with pulmonary arterial hypertension treated with first-line bosentan. Eur J Clin Invest. 2006;36 Suppl 3:10-15.
39. Denton CP, Humbert M, Rubin L, Black CM. Bosentan treatment for pulmonary arterial hypertension related to connective tissue disease: a subgroup analysis of the pivotal clinical trials and their open-label extensions. Ann Rheum Dis. 2006;65:1336-1340.

40. Gatzoulis MA, Beghetti M, Galiè N, et al. Longer-term bosentan therapy improves functional capacity in Eisenmenger syndrome: Results of the BREATHE-5 open-label extension study. Int J Cardiol. 2008; 127:27-32.

41. Frost AE, Langleben D, Oudiz R, et al. The 6-min walk test (6MW) as an efficacy endpoint in pulmonary arterial hypertension clinical trials: demonstration of a ceiling effect. Vascul Pharmacol. 2005;43: 36-39.

42. Galiè N, Ghofrani HA, Torbicki A, et al; for the Sildenafil Use in Pulmonary Arterial Hypertension (SUPER) Study Group. Sildenafil citrate therapy for pulmonary arterial hypertension. $N$ Engl J Med. 2005;353:2148-2157.

43. Galiè N, Badesch D, Oudiz R, et al. Ambrisentan therapy for pulmonary arterial hypertension. J Am Coll Cardiol. 2005;46:529-535.

44. Galiè N, Olschewski H, Oudiz RJ, et al. Ambrisentan for the treatment of pulmonary arterial hypertension: results of the ambrisentan in pulmonary arterial hypertension, randomized double-blind, placebocontrolled, multicenter, efficacy (ARIES) study 1 and 2. Circulation. 2008; 117:3010-3019.

45. Simonneau G, Barst RJ, Galiè N, et al. Continuous subcutaneous infusion of treprostinil, a prostacyclin analogue, in patients with pulmonary arterial hypertension. A double-blind, randomized, placebo-controlled trial. Am J Respir Crit Care Med. 2002;165:800-804.

46. Galiè N, Humbert M, Vachiéry JL, et al; for Arterial Pulmonary Hypertension and Beraprost European (ALPHABET) Study Group. Effects of beraprost sodium, an oral prostacyclin analogue, in patients with pulmonary arterial hypertension: a randomized, double-blind, placebo-controlled trial. J Am Coll Cardiol. 2002;39:1496-1502.

47. Barst RJ, McGoon M, McLaughlin V, et al; for Beraprost Study Group. Beraprost therapy for pulmonary arterial hypertension. $\mathrm{J} \mathrm{Am} \mathrm{Coll}$ Cardiol. 2003;41:2119-2125.

48. Barst RJ, Langleben D, Frost A, et al; for STRIDE-1 Study Group. Sitaxsentan therapy for pulmonary arterial hypertension. Am J Respir Crit Care Med. 2004;169:441-447.

49. Sastry BKS, Narasimhan C, Reddy NK, Raju BS. Clinical efficacy of sildenafil in primary pulmonary hypertension: A randomized, placebo-controlled, double- blind, crossover study. J Am Coll Cardiol. 2004;43:1149-1153.

50. Simonneau G, Rubin L, Galiè N, et al; for the Pulmonary Arterial Hypertension combination Study of Epoprostenol Sildenafil (PACES) Study Group. Addition of sildenafil to long-term intravenous epoprostenol therapy in patients with pulmonary arterial hypertension. Ann Intern Med. 2008;149:521-530.

51. Torres F. Long-term ambrisentan therapy in patients with pulmonary arterial hypertension: an analysis by WHO Functional Class [abstract]. Am J Respir Crit Care Med. 2009;179. A3370.

52. Hoeper MM, Faulenbach C, Golpon H, Winkler J, Welte T, Niedermeyer J. Combination therapy with bosentan and sildenafil in idiopathic pulmonary arterial hypertension. Eur Respir J. 2004;24:1007-1010.

53. Sitbon O, McLaughlin VV, Badesch DB, et al. Survival in patients with class III idiopathic pulmonary arterial hypertension treated with first line oral bosentan compared with an historical cohort of patients started on intravenous epoprostenol. Thorax. 2005;60: 1025-1030.

54. Benza RL, Frost A, Girgis R, Langleben D, Lawrence EC, Naeije R. Chronic treatment of pulmonary arterial hypertension (PAH) with sitaxentan and bosentan [abstract]. Proc Am Thorac Soc. 2006;3:A729.

55. Highland KB, Strange C, Girgis RE, Black C. Comparison of sitaxentan and bosentan in pulmonary arterial hypertension associated with connective tissue disease [abstract]. Ann Rheum Dis. 2006;65 Suppl 2:393.

56. No author listed. Tracleer (bosentan tablets) 62.5 and $125 \mathrm{mg}$ film-coated tablets. http://www.tracleer.com/pdf/01 00101090309 Tra_PromoPI_ SinglePages_TR4385_030409_FINAL.pdf. Accessed April 2009. 
57. Segal S. Travcleer_dhcp_final.pdf[Letter dated 2006 March 1]. United States Food and Drug Administration [safety information pages]. Silver Spring [updated 2009 June 19; cited 2009 June 19]. http://www.fda.gov/Safety/ MedWatch/SafetyInformation/SafetyAlertsforHumanMedicalProducts/ ucm150762.htm. Accessed April 2009.

58. Dwyer N, Jones G, Kilpatrick D. Severe hepatotoxicity in a patient on bosentan upon addition of methotrexate: reversible with resumption of methotrexate without bosentan. J Clin Rheumatol. 2009;15:88-89.
59. Galiè N, Manes A, Negro L, Palazzini M, Bacchi-Reggiani ML, Branzi A. A meta-analysis of randomized controlled trials in pulmonary arterial hypertension. Eur Heart J. 2009;30:394-403.

\section{Publish your work in this journal}

Vascular Health and Risk Management is an international, peerreviewed journal of therapeutics and risk management, focusing on concise rapid reporting of clinical studies on the processes involved in the maintenance of vascular health; the monitoring, prevention and treatment of vascular disease and its sequelae; and the involvement of metabolic disorders, particularly diabetes. This journal is indexed on PubMed Central and MedLine. The manuscript management system is completely online and includes a very quick and fair peer-review system, which is all easy to use. Visit http://www.dovepress.com/ testimonials.php to read real quotes from published authors.

Submit your manuscript here: http://www.dovepress.com/vascular-health-and-risk-management-journal 